\title{
Integrated Mobility: A Research in Progress
}

\author{
Gianmario Motta ${ }^{1}$, Antonella Ferrara ${ }^{1}$, Daniele Sacco ${ }^{1}$, Linlin You ${ }^{1}$, Gianpaolo Cugola ${ }^{2}$ \\ ${ }^{1}$ Dept. of Industrial and Information Engineering, University of Pavia, Pavia, Italy; ${ }^{2}$ Dept. of Electronic and Information Engineering, \\ Politecnico of Milan, Milan, Italy.
}

Received 2013

\begin{abstract}
We here present an ongoing research project about an integrated real time mobility assistant, which has been developed for the call "Personal Integrated Mobility" of the EU framework program. The assistant is aimed to support personal mobility in a near future scenario, where green, shared or public transports are replacing the current private, carbon transportation system. The assistant handles itineraries, which are based on goals of time, energy/pollution and cost, and supports users both before and during the trip. The service will gather and interpret any relevant source of information on transport resources and their availability. Information includes user generated content and social data. Given the globalization of users and the hourly peaks of mobility, the assistant is a cloud service, which integrates existing technologies; however a global extension requires a federation of clouds and, above all, the interpretation and validation of social data. These can be considered major research challenges.
\end{abstract}

Keywords: Smart Cities; Cloud Computing; Mobility Management; Mobility Integrator; Service System

\section{Introduction: The Personal Mobility Isssue}

The world population is increasingly city-based. First most people live in cities: 3.6 billion nowadays and in $205068 \%$ of the world population [1]. Second, cities consume $80 \%$ of worldwide energy production and generate $67 \%$ of energy-related greenhouse gases [2]. Third, $64 \%$ of travel kilometers are urban and the travel within urban areas is expected to triple by 2050 [3]. Hence, urban mobility is a challenge.

In 2011 the mobility maturity of 66 cities worldwide was assessed by 11 criteria [4], ranging from public transport share to average travel speed and transport-related C02 emissions. With a mobility score from 0 to $100 \mathrm{EU}$ had the best regional performance with an average of 71.4 points, with seven out of the 18 analyzed cities scoring above 75 points, while Eastern/South Eastern Europe performed a regional average of 64.0 points. Also Asian cities e.g. Hong Kong, Singapore and Shanghai reach the high and middle-high level [4]. Smart city projects are focusing on environment issues. Amsterdam Smart City [6], is a cradle for twenty pilot projects developed in cooperation with 72 partners aimed at reducing emissions of carbon dioxide. Lyon [7] carries on an integrated mobility project for people, travelers and freights, starting in 2012, with the goal of reducing emissions. In Portugal, MOBI.E [8] is a modeling framework for smart mobility, includes a network for sharing electrical vehicles. In Malaga [9] a project is deploying a fleet of electrical vehicles. All these projects intend to reduce emissions and energy saving, with a positive social and economic impact $[10,11]$.

What shall be a future ideal scenario? According to Horizon 2020 [5] in future smart cities transport shall be smart, green, and integrated. To support an intelligent mobility, cities have to get more and more wired and the smart phone users have to grow from the current 55-60\% to $100 \%$ of mobile phones. This, with the increasing number of smart terminals such as smart TV and in-vehicle devices, will enable users to be served by an integrated mobility information service [12].

However, a future, ideal scenario requires also a new mobility service concept. Mobility Integrator is an innovative approach that integrates Information \& Planning, Transport Services, Infrastructure and Traffic Management. In short, it shall provide a smooth and convenient integrated mobility platform. Let us mention some projects that fall within these objectives.

Instant Mobility [13] intends to manage mobility for different stakeholders, based on future internet infrastructure (FI-WARE). It enables travelers to view realtime traffic status and public transport availability and optimize their routes according to current personal preferences and constraints. The service also supports local authorities, public transport operators and professional drivers to optimize traffic and promote car sharing and pooling. In addition, logistic is enhanced and optimized for fleet management. 
TRIPZOOM [14] implements a new approach to urban mobility by sharing personal mobility patterns via social networks. The research \& development project SUNSET is part of the European Commission's Seventh Framework program Smart Cities \& Sustainability (DG Connect) and consists of 9 partners from 4 European countries. It encourages citizens to use sustainable forms of transportation and intends to generate a win-win game for all involved stakeholders. E.g. citizens can optimize their mobility needs using recommendation and personalized traffic services from the city authority. The city authority can accurately assess current infrastructure use and induce mobility patterns by an incentive system. Third-party service providers can tap into the data to create new offerings and integrate with others through common incentive structures. Generally, TRIPZOOM focuses on sharing trip information on social networks and optimizing the personal mobility according to incentives and suggestions derived by mobility sensing. It can be seen as an attempt to integrate Traffic Management and Information and Planning.

In Future Urban Mobility program [15] developed by the Massachusetts Institute of Technology (MIT) and the National Research Foundation of Singapore, SimMobility model is designed to use personal mobile computing and communication devices to provide high quality information about the state of the transportation network to users and system managers, working as data collectors and computing engines. The main objective of SimMobility is to guarantee the promise those urban citizen demands, such as energy consumption and transportation flows are derived from human needs. Currently, only a framework has been developed. The intent is to create a model that serves as a decision-supporting tool for urban planners and policy makers. It gathers, processes, stores and shares the data from different sources including personal devices, but it is mainly focused on traffic management in the urban area.

\section{An Integrated Mobility Assistant}

Our mobility proposal, called IRMA (Integrated Realtime Mobility Assistant), targets individuals in whole lifecycle of their mobility. Work started in Department of Information and Industrial Engineering of Pavia University to develop an App that could assist travelers to meet their schedule even with of transport disruptions [16]. From further investigations emerged a need of a more comprehensive architecture. First, the mobility App had to be able to process any useful information source; second, it had to be universal and usable in any urban area, thus implying a Service Oriented Architecture in a cloud environment. Such integrated architecture, which has defined for call 6.6 of the European Framework Program, called "Personal Integrated Mobility", is presented here.
Here below illustrate first the functional concept.

IRMA handles itineraries, performed by one person or a group, that are conceived as an oriented graph [17], whose connections are represented by nodes, while energy or pollution requirements are modeled as resource constrains as it happens with project modeling techniques $[18,19]$. Finally, itinerary cost is considered as third variable. Itinerary is a master information that is integrated both before and during the trip, by additional information about:

- Transport map, that records transport resources which are / will be available e.g. train or underground;

- Transport status, that records future and current availability and delay; e.g. train timetable, train delay, underground load, road traffic and deviation from standard travel time;

- Relevant information from social network, that is handled as a text message (traffic jams, road bumps etc.)

The processing cycle of IRMA conceptually is a Big Data version [21,22] of the classic BI schema [20], that adds semantic interpretation/ transformation of social information [23,24]. In short, IRMA aggregates a network of information sources, typically spread in clouds, to a personal information service. In Table 1 we summarize the functional coverage of IRMA versus the before mentioned Instant Mobility (IM) TRIPZOOM (TZ) and Future Urban Mobility (FUM). The table lists main features of integrated mobility solutions, namely the stakeholders which are targeted, the data sources that are involved, and, finally, the services that are provided to the individual users. The focus of IRMA are the services to the individual mobile users. IRMA manages the whole individual mobility lifecycle of an itinerary, which goes from A to B through multiple connections. Such itinerary can be analyzed, forecasted and assisted using historical and real-time data- More than that, mobility users can get more effective and efficient assistants to manage their itineraries by two dimensions in terms of before the trip and after the trip. IRMA is a cloud-based solution for personal mobility. The services are built upon a Future Internet-based infrastructure by which various data sources are integrated. Upon that infrastructure, different stakeholders' views are served: users, transport providers and municipalities.

\subsection{The Overall Architecture}

IRMA architecture includes various elements, namely (a) mobility analysis (b) mobility forecasting (c) mobility assistant (d) terminals (e) communication services (f) sources which is shown in Figure 1. Let us shortly describe these elements. 
Table 1. Functional coverage of IRMA: a comparison.

\begin{tabular}{lllll}
\hline \multirow{2}{*}{ Features } & & \multicolumn{3}{c}{ Projects } \\
\cline { 3 - 4 } Stake & Mobility User & & & \\
\cline { 3 - 4 } holders & Municipality & TZ & FUM & IRMA \\
& Transport Provider & & & \\
& Transportation & & \\
& Management Systems & & \\
Tata Sources & Traffic Management & & \\
& Systems & & \\
& Vehicles & & \\
& Social & & \\
& Networks & & \\
& Mobility analysis & & Part \\
Services & Individual Mobility & & \\
& Planning & & \\
\hline & Individual real-time & & \\
\hline
\end{tabular}

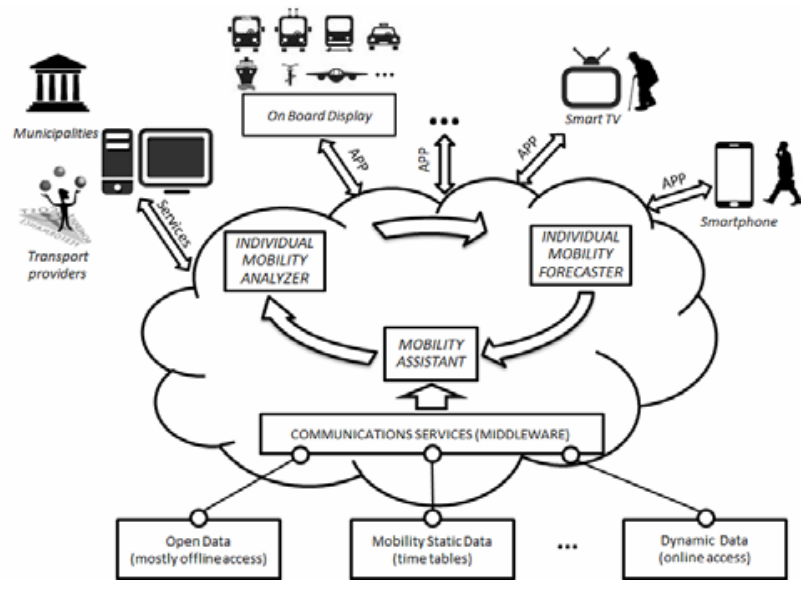

Figure 1. IRMA Architecture.

\subsection{The Individual Mobility Analyzer}

It stores a mobility map and related mobility data. The mobility map describes mobility resources within the urban area, by route, time, and mode. In turn mobility data describe the mobility load by route, time and date. Users can analyze mobility across any urban area, while municipalities and transport enterprises can analyze actual versus expected mobility performances. These historical data are an input to the individual mobility forecaster.

\subsection{The Individual Mobility Forecaster}

We assume that the urban area is divided in cells. The forecast formulates a discrete event framework that gives the ability to anticipate the effects on the itinerary by predicted or unpredicted future events (e.g. football match, exhibition, holiday, accident etc.). Forecasted travel duration is an input to the mobility assistant, where the user can choose the best route, taking into account the dynamics of transportation modes and user constrains.

\subsection{The Personal Mobility Assistant}

It shall assist the end user to plan, configure, monitor, alarm, and reschedule mobility across multiple mobility options. It manages and supports the mobility itinerary by two phases in the mobility life cycle with different modules/services.

Before the trip (Planning). Request Handler processes the mobility request of each user. Request may concern an individual trip or a mobility calendar. It helps the user to define the optimal mobility plan by accessing mobility forecast and mobility maps through the Information Retrieval sub service. The user will choose and confirm the ideal option as an individual itinerary. In the Figure 2, you see simplified screens where transport options that are sorted by time or by energy efficiency. Transport time is based on the forecaster that adjusts the standard time on the traffic load projections related to transport modes, the specific daytime, date, and the route from A to B.

During the trip. During the trip the user receives information about disruptions (Event Notifier) and use the assistance to choose a viable alternative (Compensation Engine). An informal representation of the user's view is in Figure 3. Event Notifier is a set of instances that are activated when the user confirms and actually starts the trip. It concerns all connections of the individual itinerary and process relevant disruption information provided by communication services. Compensation Engine processes mobility alternatives in front of a disruption or a change, by browsing on mobility analyzer the closest option (as an alternative the request handler could fetch a plan B in advance).

\subsection{Terminals}

IRMA shall be used by a variety of mobile and fixed terminals, which include smart phone/tablet/ laptop

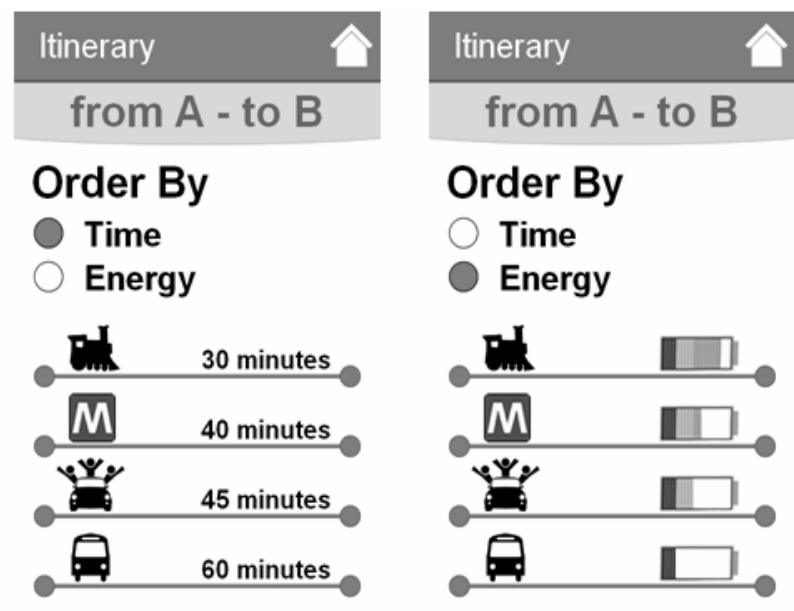

Figure 2. Before a trip - Planning a trip - illustrative screens. 


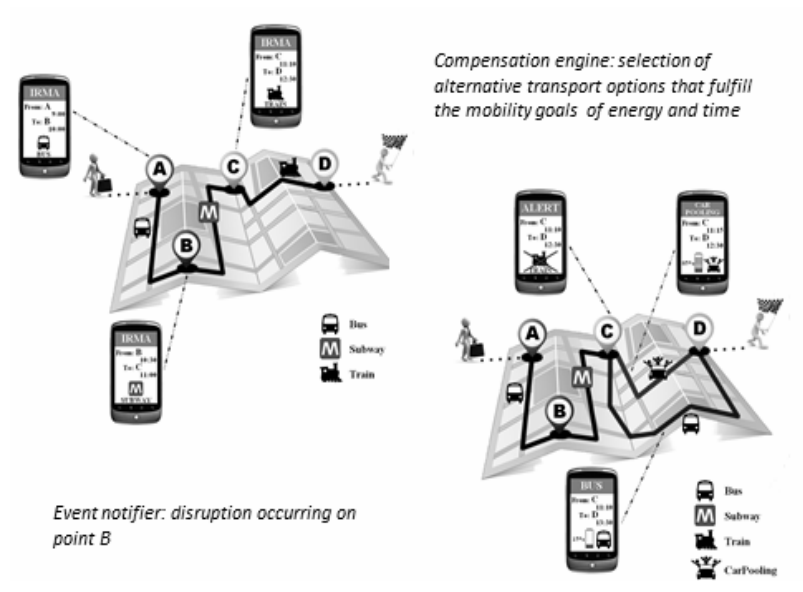

Figure 3. During the trip - Reschedule a trip - illustrative screens.

computers, smart TV to support ageing people, web applications on board displays, etc.

\section{Integrating the Mobility Assistant with Information Sources}

A SOA - EDA (Service Oriented Architecture - Event Driven Architecture) infrastructure enables services that access field information on mobility. Such collection involves various data sources. Mobility data include time tables \& delays of planes, trains, buses, underground; availability of taxi, carpooling, parking. Traffic data encompass data gathered from traffic \& environment management systems. In turn, vehicle data include data on mobility from bus, trains, cars, bikes etc. Finally, crowd data comprise alarms and messages refined by ad hoc schema coming from Twitter, Facebook, YouTube, etc.

Through a communication layer, the system gets information on traffic and mobility, with various purposes, e.g. access to historical data, on demand access, real-time access. The access on historical mobility data is intended to collect historical mobility information that will be processed by Analyzer and Forecaster modules; sources may include traffic systems and transport systems. On demand access concerns information about availability of transport resources e.g. trains, urban public transportation, carpooling, bikes. Finally, real-time access gets information to moving users; information is taken on the delay or disruption that happens while users are moving. Sources are data and services of the systems the manage mobility, namely transport operators, municipalities, mobility users, that have been mentioned before. The itineraries of IRMA users may be also a statistical source, if data are cleaned and made anonymous in order to protect privacy.

\section{Conclusion}

We have illustrated the key concepts IRMA, i.e. an inte- grated mobility assistant, which we regard as a stage 2 of smart cities. It is in a design in progress and only small lab demonstrators have been made. Generally, IRMA looks feasible, because it mainly integrates existing technologies; however, it goes beyond the state of the art on several aspects, among which data integration as a key one. Let us consider this issue.

IRMA uses heterogonous information from multiple sources. Hence, the correspondences among similar entities (instances and concepts) in different datasets have to be found in order to make a virtually integrated knowledge base available. Tis implies to develop scalable methods to select, triplify, match, and integrate mobilityrelated open data and user generated content, so that these data can be plugged in the data warehouse of the analytic IRMA services. Two research directions will be explored, as efficient techniques for the on-the-go matching of linked data schemas and quality-driven methods to fuse semantic data coming from different sources will be defined by extending techniques applied to service descriptions extracted from Web sources [27], or by adopting a Human Computation approach to improve urban-related information via user-centric contributions [28].

IRMA is expected to impact on environment, business and users. As far as environment is concerned, the service will lower energy and emissions, since it will enable to use public/shared transport systems even with complex connections. Also, a variety of stakeholders can benefit from IRMA. In business perspective, municipalities and transport providers can analyze and forecast mobility in terms of time, route, connection, and mode, and, therefore, can optimize transportation resources. Finally, mobile users can define their travel and can actually travel in an optimal way, without any previous knowledge of any transportation system.

Finally, as future goal, with a cloud based service as IRMA, users will, ideally, plan their mobility in any urban area across the world. Also, a global coverage implies several advances in clouds, as interoperability among cloud offerings with federating applications running on different clouds [25], and combine pull and push approaches by integrating Service Oriented and Event Driven architectures. Finally, IRMA can be platform for a global virtual ticket. Simply, the users buy a mobility ticket, that is nothing else but a credit, alike a SIM recharge, that can be spent on the itineraries managed by IRMA.

\section{REFERENCES}

[1] UN, World Urbanization Prospects: The 2011 Revision. UN: New York. 2012

[2] Hoornweg, D., Freire, M., Lee, M. J., Bhada-Tata, P., \& 
Yuen, B. (Eds.). (2011). Cities and climate change: responding to an urgent agenda. World Bank Publications

[3] Dixon, T. (2011). Sustainable Urban Development to 2050: Complex Transitions in the Built Environment of Cities. WP2011/5 October

[4] Lerner, W., \& Van Audenhove, F. J. (2012). The future of urban mobility: Towards networked, multimodal cities in 2050. Public Transport International-English Edition, 61(2), 14

[5] Horizon 2020: http://ec.europa.eu/research/horizon2020/

[6] Amsterdam Smart City web site: amsterdamsmartcity.com

[7] Greater Lyon project web site: www.business.greaterlyon.com/lyon-smart-city-france-eu rope.346.0.html?\&L=1

[8] MOBI.E web site: www.mobie.pt

[9] Malaga Smart Mobility Project web site: www.smartcitymalaga.es

[10] De Nicolao G., Ferrara A., \& Giacomini L. (2007). On board sensor-based collision risk assessment to improve pedestrians' safety, IEEE Transaction on Vehicular Technology, 56, 5, 1, (pp. 2405 - 2413)

[11] Ferrara A., \& Vecchio C. (2008). Second order sliding mode control of a platoon of vehicles, Int. Journal of Modelling, Identification and Control, 3, 3

[12] http://mobithinking.com/mobile-marketing-tools/latest-m obile-stats/a\#smartphone-shipments

[13] FI-PPP Instant Mobility website: www.instant-mobility.org

[14] Holleis, P., Luther, M., Broll, G., Hu, C., Koolwaaij, J., Peddemors, A., \& Raaphorst, S. (2012, May). TRIPZOOM: a System to Motivate Sustainable Urban Mobility. In SMART 2012, The First International Conference on Smart Systems, Devices and Technologies (pp. 101-104)

[15] Ben-Akiva, Moshe E. (2010, November), SMART - Future Urban Mobility, JOURNEYS Sharing Urban Transport Solutions, 5 (pp. 30-37)

[16] Motta G., Barrorero T., Telese F. , Design of performance aware service systems, The International Joint Conference on Service Sciences (IJCSS) 2011, Taiwan

[17] Berge, C., Théorie des graphes et ses applications, Collection Universitaire de Mathématiques, II, Paris: Dunod,
1958

[18] Fazar, W., "Program Evaluation and Review Technique", The American Statistician, Vol. 13, No. 2, (April 1959), p.10.

[19] Kelley, James; Walker, Morgan. Critical-Path Planning and Scheduling. 1959 Proceedings of the Eastern Joint Computer Conference.

[20] Inmon, William H., et al. Building the data warehouse. J. Wiley, 2002; first edition (1992).

[21] Gorton, I., Greenfield, P., Szalay, A., \& Williams, R. (2008). Data-intensive computing in the 21st century. Computer, 41(4), 30-32.

[22] Agrawal, D., Das, S., \& El Abbadi, A. (2011, March). Big data and cloud computing: current state and future opportunities. In Proceedings of the 14th International Conference on Extending Database Technology (pp. 530-533). ACM.

[23] Tutorial on Information value and conceptual modelling: a systemic survey of economic, social and technological issues, ER Conference 2012, Florence, Italy, available on line (three pdf documents) at http://islab.dico.unimi.it/er2012/index.php/tutorials.

[24] S. Morris, H. Shin - Social Value of Public Information, The American Economic Review, Volume 92, Number 5, 1, 2002.

[25] D. Ardagna, E. Di Nitto, D. Petcu, P. Mohagheghi, S. Mosser, P. Matthews, A. Gericke, C. Ballagny, F. D'Andria, C. Nechifor, C. Sheridan. MODACLOUDS: A Model-Driven Approach for the Design and Execution of Applications on Multiple Clouds. MiSE 2012 Workshops Proceedings.

[26] C. Batini, C. Cappiello, C. Francalanci, A. Maurino, G. Viscusi: A capacity and value based model for data architectures adopting integration technologies. AMCIS 2011.

[27] I. Celino, S. Contessa, M. Corubolo, D. Dell'Aglio, E. Della Valle, S. Fumeo, T. Krüger: Linking Smart Cities Datasets with Human Computation - The Case of UrbanMatch. Int. Semantic Web Conf. (2) 2012

[28] L. Panziera, M. Comerio, M. Palmonari, F. De Paoli, C. Batini: Quality-driven Extraction, Fusion and Matchmaking of Semantic Web API Descriptions. J. Web Eng. 11(3): 247-268 (2012). 